\title{
Levantamento da disponibilidade de dados hidrométricos na Paraíba - Brasil
}

\author{
Survey of Hidrometric Data Availability in Paraíba - Brazil \\ Katherine da Silva Sousal, Iury Araujo Macêdo Dantas², Gabriela Braga de Sá3, Anderson Bruno Anacleto de Andrade \\ Patrício Borges Maracajá ${ }^{5}$
}

Resumo: A limitação dos recursos hídricos na atualidade é um dos principais entraves para o desenvolvimento econômico e social, e leva a muitos desafios para o planejamento e gerenciamento deste recurso. O levantamento das informações e dados necessários para elaboração de estudos hidrológicos é realizado através de estações hidrométricas, cujas séries de dados têm sua importância proporcional à sua extensão temporal. O presente estudo objetiva elaborar um levantamento das estações pluviométricas e fluviométricas com existência e disponibilidade de dados de chuva e vazão das bacias hidrográficas do estado da Paraíba. O levantamento foi elaborado junto à Rede Hidrometeorológica Nacional da Agência Nacional de Águas - ANA, obtidos a partir seu portal Hidroweb, onde são disponibilizados os dados hidrológicos em séries históricas. Foram identificadas 79 estações pluviométricas e 20 estações fluviométricas com dados existentes na Paraíba, após esse levantamento foi elaborado um mapa temático para visualização as estações que correspondem a cada bacia do estado da Paraíba, a fim de auxiliar na análise dos resultados, onde mostrou que as estações hidrométricas se encontram distribuídas de forma diversificada nas 11 bacias hidrográficas do estado, apresentando bacias sem dados de chuva e vazão disponíveis.

Palavras-chaves: Precipitação, vazão, estações pluviométricas, estações fluviométricas.

Abstract: The limitation of water resources at the present time is a major constraint to economic and social development, and leads to many challenges to the planning and management of this resource. The survey of information and data required for the preparation of hydrological studies are conducted through hydrometric stations whose data series have their proportional importance to its temporal extension. This study aims to draw up a survey of rainfall and gauged stations with existence and availability of rainfall data and flow of river basins of the state of Paraíba. The survey was prepared by the National Hydrometeorological Network of the National Water Agency - ANA, obtained from its website Hidroweb where hydrological data in time series are available. 79 were identified rainfall stations and 20 gauged stations with data in Paraíba, after this survey was prepared a thematic map to view the stations corresponding to each state of Paraiba Basin in order to assist in the analysis of the results, which showed that hydrometric stations are distributed in a diversified way in the 11 watersheds status showing no bowls available rainfall and flow data.

Key words: Rainfall, flow, rainfall stations, gauged stations.

\footnotetext{
*Autor para correspondência

Recebido para publicação em 02/03/2015; aprovado em 27/03/2015

${ }^{1}$ Mestranda em Sistemas Agroindustriais, Universidade Federal de Campina Grande, Pombal-PB; (83) 99619-5586, E-mail:katherinesousasilva@hotmail.com.

${ }^{2}$ M. Sc. Sistemas Agroindustriais, Graduado em Engenharia Ambiental, Universidade Federal de Campina Grande, E-mail: iury.araujo@hotmail.com.

${ }^{3}$ Graduanda em Engenharia Ambiental, Universidade Federal de Campina Grande, E-mail: gabrielasa.1@ @otmail.com.

${ }^{4}$ Graduando em Agronomia , Universidade Federal de Campina Grande. E-mail: bdeandrade3@gmail.com

${ }^{5}$ D. Sc. Prof. Associado IV. Curso de Pós graduação Stricto Sensu em Sistemas Agroindustriais do CCTA/UFCG. E-mail: patriciomaracaja@ gmail.com
} 


\section{INTRODUÇÃO}

A limitação dos recursos hídricos na atualidade é um importante condicionante ao desenvolvimento econômico e social e acarreta inúmeros desafios ao planejamento e gerenciamento deste recurso.

O conhecimento do regime hidrológico no estado da Paraíba e sua variabilidade são de grande importância para planejamento de ações emergenciais, uma vez que o estado localiza-se em uma região suscetível a deficiências hídricas para o planejamento (GOMES et al, 2015; BECKER et al, 2013).

O estudo da hidrologia compreende a coleta de dados básicos, sendo a quantidade de água precipitada e a vazão dos rios variáveis fundamentais para a elaboração de estudos, a partir da analise desses dados pode-se estabelecer suas relações mútuas e o entendimento da influência de cada fator e aplicar os conhecimentos obtidos para solução de inúmeros problemas práticos (PINTO et al, 1976).

$\mathrm{O}$ levantamento das informações necessárias à hidrologia é realizado através de redes de estações hidrométricas, cujas séries de dados têm sua importância proporcional à sua extensão temporal (COSTA \& FERNANDES， 2015; SILVEIRA， 2010; ANA， 2009; GALINA \& VERONA, 2004).

A lei Federal 9433/97, que instituiu a política e o Sistema Nacional de Gerenciamento de Recursos Hídricos, conhecida também como a lei das águas, cria instrumentos para efetivar a gestão dos recursos hídricos na bacia hidrográfica, onde um dos seus instrumentos é o sistema de informações sobre recursos hídricos.

A Agência Nacional de Águas - ANA, criada em 17 de julho de 2000, e instituída em 19 de dezembro do mesmo ano, possui dentre suas atribuições, a de promover a coordenação das atividades desenvolvidas no âmbito da Rede Hidrometeorológica Nacional, em articulação com os órgãos e entidades públicas e privadas que a integram, ou que dela sejam usuárias, cabendo então, à ANA, manter e disponibilizar o cadastro atualizado das estações hidrometeorológicas do País (ANA, 2009). Assim como disponibilizar informações e dados das estações hidrométricas nacionais a fim de servir de suporte a estudos hidrológicos.

A Resolução Conjunta ANA ANEEL n ${ }^{\circ}$ 03, de 10 de agosto de 2010, publicada em 20 de outubro de 2010, determina a ANA a função de orientar os agentes do setor elétrico sobre os procedimentos de coleta, tratamento e armazenamento dos dados hidrométricos objetos do normativo, bem como sobre a forma de envio dessas informações em formato compatível com o Sistema Nacional de Informações sobre Recursos Hídricos (SNIRH), o que permitirá a difusão dos dados em "tempo real" oriundos do monitoramento hidrológico realizado pelos agentes do setor elétrico (ANA, 2012; ANA/ANEEL, 2009).

Os estudos hidrológicos auxiliam os gestores e os tomadores de decisão com informações que contribuem para a garantia da oferta de água, em quantidade e qualidade suficientes para os usos múltiplos (ELESBON et al., 2014; ANA, 2012; TUCCI, 2002).

Deve-se destacar que a qualidade dos dados hidrológicos é essencial em qualquer estudo, sendo necessário realizar uma análise da qualidade dos dados por meio da identificação prévia dos postos que permite a utilização de dados de postos que possuam dados confiáveis. Os postos devem ser classificados conforme a confiabilidade das informações que dispõe, a fim de permitir que o usuário melhor defina a análise regional (CALDEIRA et al, 2015; VILANOVA, 2013; TUCCI, 2002).

Os dados hidrológicos são medidos em locais definidos, como um pluviômetro numa bacia, que observa a ocorrência de precipitação numa amostra pontual, e um posto fluviométrico numa seção de um rio, que observa o escoamento na bacia hidrográfica em forma de vazão.

Os dados fluviométricos e pluviométricos são indispensáveis para os estudos de aproveitamentos hidroenergéticos, planejamento de uso dos recursos hídricos, previsão de cheias, gerenciamento de bacias hidrográficas, saneamento básico, abastecimento público e industrial, navegação, irrigação, transporte, meio ambiente e muitos outros estudos de grande importância científica e sócioeconômica (LEAL, 2012; LUERCE et al., 2011; IBIAPINA et al. 1999).

O presente estudo objetiva elaborar um levantamento das estações pluviométricas e fluviométricas com existência e disponibilidade de dados de chuva e vazão das bacias hidrográficas do estado da Paraíba.

\section{MATERIAL E MÉTODOS}

\section{Área de estudo}

Os procedimentos desenvolvidos nesta pesquisa foram aplicados ao estado da Paraíba, inserido na região Nordeste do Brasil, que possui área de $56.469,74 \mathrm{~km}^{2}$ e população de 3.943.885 milhões de habitantes (IBGE, 2010).

No estado da Paraíba estão inseridas 11 bacias hidrográficas, conforme mostra a Figura 1.

Figura 1 - Bacias hidrográficas do estado da Paraíba

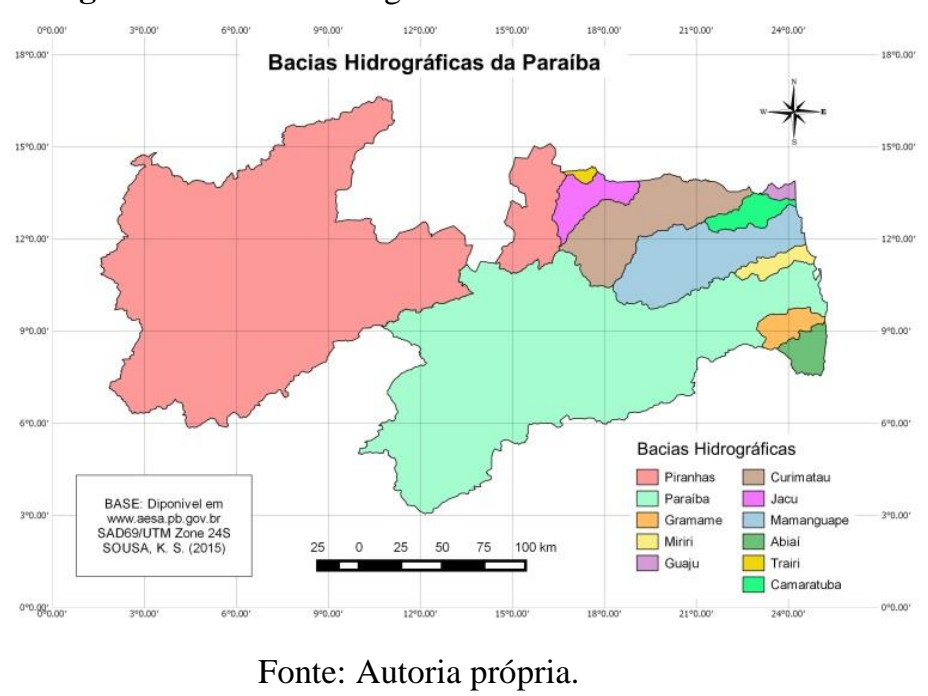

Compreende com as coordenadas geográficas: $07^{\circ} 09^{\prime} \mathrm{S}$ e $36^{\circ} 49^{\prime}$ W. Limita-se ao norte com o estado do Rio Grande do Norte (norte), ao sul com Pernambuco e a oeste com o Ceará. Drenando uma área de aproximadamente 56.469, 778 $\mathrm{km}^{2}$, área referente a todo o território estadual.

No Estado da Paraíba destacam-se quatro ecossistemas naturais principais, Planícies Litorâneas, de Florestas, de Áreas em Transição e de Caatinga. Possui relevo bastante 
diversificado, constituindo-se por formas de relevo diferentes, onde foram trabalhadas por diferentes processos, atuando sob climas distintos e sobre rochas pouco ou muito diferenciadas. A geomorfologia da Paraíba é dividida em dois grupos compreendidos pelos tipos climáticos mais significativos do Estado: úmido, sub-úmido e semiárido. O estado localiza-se numa faixa equatorial, com alta incidência de radiação solar, com temperatura média anual de $26^{\circ} \mathrm{C}$ (PARAÍBA, 2006).

\section{Levantamento de dados hidrométricos}

O levantamento das estações pluviométricas e fluviométricos com dados existentes foram elaborados por meio da Rede Hidrometeorológica Nacional da Agência Nacional de Águas - ANA, obtidos a partir do ao portal Hidroweb, onde são disponibilizados os dados hidrológicos em séries históricas.

Os dados disponibilizados apresentam informações diárias e mensais das estações pluviométricas e fluviométricas, disponíveis por região, desde a data de inicio do funcionamento.

Cada estação apresenta um período de funcionamento diferente, tornando necessária a organização dos arquivos de forma individual.

Elaboração de mapa temático
Após levantamento das estações pluviométricas e fluviométricas com dados existentes e disponíveis, foi elaborado um mapa temático para visualização as estações que correspondem a cada bacia do estado da Paraíba, a fim de auxiliar na análise dos resultados.

Para confeccionar o mapa com os pontos coletados, foi utilizando a versão 2.6.0 do programa QSIG, e convertendo, inicialmente, os dados contidos em arquivo do Excel (formato CVC) em um arquivo vetorial (tipo Shapefile- SHP). Em seguida, converteu-se o arquivo vetorial que se encontrava na projeção WGS843 para a projeção SAD69/UTM Zone 24S.

As coordenadas coletadas junto ao portal Hidroweb da ANA foram adicionadas ao mapa de bacias hidrográficas da Paraíba, elaborando assim, um mapa com os postos pluviométricos e fluviométricos com dados existentes e disponibilizados.

\section{RESULTADOS E DISCUSSÃO}

\section{Levantamento de dados hidrométricos}

Foram identificadas 368 estações pluviométricas e 206 estações fluviométricas no estado da Paraíba, sendo 79 pluviométricas e 20 fluviométricas com existência e disponibilidade de dados.

As estações hidrométricas com disponibilidade de dados de chuva e vazão estão apresentadas nos Quadros 1 e 2 .

Quadro 1 - Estações pluviométricas com dados disponibilizados

\begin{tabular}{|c|c|c|c|c|c|}
\hline Código & Estação & Período de dados & Código & Estação & Período de dados \\
\hline 635027 & Araçagi & 1962 a 1994 & 735012 & Fazenda Lagoa dos Marcos & 1962 a 1994 \\
\hline 635028 & Araruna & 1911 a 1994 & 735015 & Itabaiana & 1910 a 1994 \\
\hline 635030 & Areia & 1910 a 1994 & 735017 & Ingá & 1910 a 1994 \\
\hline 635032 & Areia $^{2}$ & 1963 a 1966 & 735018 & Fagundes & 1962 a 1993 \\
\hline 635033 & Areia I & 1971 a 1972 & 735019 & Cruz do Espírito Santo & 1911 a 1990 \\
\hline 635033 & Bananeiras & 1930 a 1994 & 735024 & Campina Grande 2 & 1962 a 1968 \\
\hline 635037 & Cacimba de Dentro & 1962 a 1994 & 735025 & Campina Grande 3 & 1963 a 1984 \\
\hline 635038 & Caiçara & 1962 a 1994 & 735026 & Campina Grande 4 & 1910 a 1985 \\
\hline 635040 & Guarabira & 1910 a 1982 & 735028 & Bodocongó 1 & 1933 a 1994 \\
\hline 635043 & Jacaraú & 1962 a 1993 & 735029 & Aroeiras & 1962 a 1994 \\
\hline 635064 & Areia II & 1971 a 1974 & 735030 & Alagoa Nova & 1911 a 1993 \\
\hline 635079 & Fazenda Alagamar & 1998 a 2014 & 735033 & Alagoa Grande & $\begin{array}{l}1910 \text { a } 1933 \\
1959 \text { a } 1990\end{array}$ \\
\hline 636022 & Cabaceiras & 1929 a 1994 & 735035 & Açau & 1962 a 1994 \\
\hline 636031 & Algodão & 1962 a 1983 & 735124 & Bodocongó & 1970 a 2014 \\
\hline 636032 & Barra de Santa Rosa & 1930 a 1983 & 736017 & Coxixola & 1962 a 1994 \\
\hline 636035 & Cuité & 1962 a 1983 & 736018 & Congo & 1962 a 1994 \\
\hline 637022 & Belém do Brejo do Cruz & 1935 a 1983 & 736019 & Catolé & 1962 a 1980 \\
\hline 637023 & Brejo do Cruz & 1931 a 1983 & 736019 & Gurjão & 1962 a 1992 \\
\hline 637024 & Cajazeirinhas & 1962 a 1981 & 736020 & Caraúbas & 1931 a 1993 \\
\hline 637025 & Catolé do Rocha & 1922 a 1979 & 736021 & Camalau & 1962 a 1994 \\
\hline 637028 & Condado & 1941 a 1983 & 736023 & Boqueirão & 1961 a 1994 \\
\hline 637029 & Desterro de Malta & 1962 a 1983 & 736024 & Boa Vista & 1923 a 1993 \\
\hline 637030 & Jenipapeiro do Cordeiro & 1962 a 1983 & 736025 & Barra de São Miguel & 1962 a 1993 \\
\hline 637036 & Jericó & 1962 a 1983 & 736026 & Fazenda Bananeiras & 1962 a 1991 \\
\hline 638015 & Balanças & 1962 a 1983 & 737017 & Imaculada & 1933 a 1993 \\
\hline 638028 & Cajazeiras & 1910 a 1983 & 737018 & Desterro & 1923 a 1993 \\
\hline 638029 & Barra do Juá & 1933 a 1983 & 737019 & Açude Coremas & 1933 a 1985 \\
\hline 638031 & Aparecida & 1962 a 1983 & 737020 & Açude Coremas - Curema & 1964 a 1977 \\
\hline 638032 & Antenor Navarro & 1984 a 2014 & 737022 & Água Branca & 1931 a 1991, \\
\hline
\end{tabular}


Katherine da Silva Sousa, et al

\begin{tabular}{|l|c|c|c|c|c|}
\hline & & & & & 1993 \\
\hline $\mathbf{6 3 8 0 3 3}$ & Antenor Navarro 2 & 1913 a 1983 & $\mathbf{7 3 8 0 0 9}$ & Fazenda Timbaúba & 1933 a 1977 \\
\hline $\mathbf{6 3 8 0 4 6}$ & Açude Pilões & 1941 a 1983 & $\mathbf{7 3 8 0 1 2}$ & Boa Ventura & 1962 a 1993 \\
\hline $\mathbf{6 3 8 0 4 7}$ & Engenheiro Ávidos & 1936 a 1983 & $\mathbf{7 3 8 0 1 7}$ & Itaporanga & 1910 a 1993 \\
\hline $\mathbf{6 3 8 0 8 8}$ & Fazenda Santo Antônio & 2008 a 2014 & $\mathbf{7 3 8 0 1 8}$ & Ibiara & 1962 a 1993 \\
\hline $\mathbf{7 0 7 3 2 1}$ & Catingueira & 1933 a 1991 & $\mathbf{7 3 8 0 1 9}$ & Garrotes & 1962 a 1993 \\
\hline $\mathbf{7 3 4 0 0 0}$ & Conde & 1962 a 1972 & $\mathbf{7 3 8 0 2 0}$ & Conceição & 1911 a 1993 \\
\hline $\mathbf{7 3 4 0 0 2}$ & João Pessoa 1 & 1964 a 1985 & $\mathbf{7 3 8 0 2 2}$ & Bonito de Santa Fé & 1933 a 1994 \\
\hline $\mathbf{7 3 4 0 0 3}$ & João Pessoa 2 & 1912 a 1985 & $\mathbf{7 3 8 0 2 3}$ & Bom Jesus & 1933 a 1991 \\
\hline $\mathbf{7 3 4 0 0 4}$ & João Pessoa 3 & 1977 a 1978 & $\mathbf{7 3 8 0 2 4}$ & Arapuã & 1935 a 1991 \\
\hline $\mathbf{7 3 4 0 0 8}$ & Alhandra & 1936 a 1994 & $\mathbf{7 3 8 0 2 5}$ & Aguiar & 1933 a 1992 \\
\hline $\mathbf{7 3 5 0 0 3}$ & Fazenda Santa Luzia & 1978 & &
\end{tabular}

Fonte: ANA (2015)

Quadro 2 - Estações fluviométricas com dados disponibilizados

\begin{tabular}{|l|l|l|l|}
\hline Código & Estação & Rio & Período de dados \\
\hline $\mathbf{3 7 2 2 0 0 0 0}$ & Várzea Grande & Rio Piranhas-Açu & 1963 a 2014 \\
\hline $\mathbf{3 7 2 3 7 0 0 0}$ & São Domingos de Pombal & Rio Piranhas-Açu & 2000 a 2007, 2013 e 2014 \\
\hline $\mathbf{3 7 2 6 0 0 0 0}$ & Antenor Navarro & Rio do Peixe & 1963 a 1973,1984 a 2014 \\
\hline $\mathbf{3 7 2 6 0 0 0 1}$ & Antenor Navarro (ex São Jão) & Rio do Peixe & 1930 a 1933,1963 \\
\hline $\mathbf{3 7 2 9 0 0 0 0}$ & Aparecida & Rio do Peixe & 1984 a 2003, 2005 a 2014 \\
\hline $\mathbf{3 7 3 4 0 0 0 0}$ & Piancó & Rio Piancó & 1963 a 2014 \\
\hline $\mathbf{3 7 3 6 0 0 0 0}$ & Emas & Rio Piancó & 1963 a 2014 \\
\hline $\mathbf{3 7 4 1 0 0 0 0}$ & Sítio Vassouras & Rio Piranhas-Açu & 1962 a 2014 \\
\hline $\mathbf{3 7 4 3 0 0 0 0}$ & Patos & Rio Espinharas & 1985 a 2007,2013 a 2014 \\
\hline $\mathbf{3 8 6 5 0 0 0 0}$ & Fazenda Alagamar & Rio Curimataú & 1981 a 2014 \\
\hline $\mathbf{3 8 7 5 0 0 0 0}$ & Mulungu & Rio Mamanguape & 1973 a 2014 \\
\hline $\mathbf{3 8 7 7 1 0 0 0}$ & Sítio Passagem & Rio Aracají & 1990 a 2014 \\
\hline $\mathbf{3 8 7 9 0 0 0 0}$ & Ponte do Leitão & Rio Mamanguape & 1970 a 2014 \\
\hline $\mathbf{3 8 7 9 2 0 0 0}$ & Ponte do Leitão - Jusante & Rio Mamanguape & 1970 \\
\hline $\mathbf{3 8 8 3 0 0 0 0}$ & Caraúbas & Rio Paraíba & 1973 a 2014 \\
\hline $\mathbf{3 8 8 5 0 0 0 0}$ & Poço de Pedra & Rio Taperoá & 1970 a 2006,2013 a 2014 \\
\hline $\mathbf{3 8 8 6 0 0 0 0}$ & Bodocongó & Rio Paraíba & 1970 a 2014 \\
\hline $\mathbf{3 8 8 8 0 0 0 0}$ & Guarita & Rio Paraíba & 1970 a 2014 \\
\hline $\mathbf{3 8 8 8 7 0 0 0}$ & Sobrado & Rio Gurinhem & 2000 a 2014 \\
\hline $\mathbf{3 8 8 9 5 0 0 0}$ & Poço da Batalha & Rio Paraíba & 1970 a 1997,2013 \\
\hline
\end{tabular}

Fonte: ANA (2015)

Os postos pluviométricos identificados possuem período de dados diferenciados, abrangendo de 1910 a 2014, ou seja, 104 anos com medições de precipitação no estado.

Pode-se observar que apenas $27,6 \%$ das estações pluviométricas possuem dados disponibilizados pela ANA e grande parte destas estações não possuem dados atualizados, apresentando dados anteriores ao ano de 2000, apenas duas possuem dados até o ano de 2014.

Quanto às estações fluviométricas, a quantidade de estações com dados disponibilizados é relativamente pequeno e pouco representativo, constando que 90,3\% das estações fluviométricas do estado da Paraíba não possuem medições de vazão ou possuem, porém não são disponibilizados.

O período de dados fluviométricos variam entre os anos de 1962 a 2014, constando de 52 anos de medições. Diferentemente das estações pluviométricas, as fluviométricas possuem dados atualizados, apresentando vazões diárias e mensais até dezembro de 2014.

Pode-se destacar também que as séries históricas de chuvas e vazão possuem falhas em seus dados, apresentando meses sem medições ao longo dos anos, sendo identificados em todas as estações hidrométricas selecionadas.
É comum encontrar-se com séries hidrológicas caracterizadas por ausência de dados, o que pode acarretar em análises errôneas e tendenciosas. Uma vez que se deseja aplicar tratamentos estatísticos em séries de precipitação, devem-se analisar os dados e identificar, caso existam, as falhas de informação durante $o$ período de interesse (CALDEIRA et al, 2015).

A ausência de registro de dados ao longo de uma série hidrológica de precipitação ocorre devido a problemas no aparelho de coleta ou então devido a erros grosseiros na observação dos dados, comprometendo assim a continuidade das informações e impossibilitando a aplicação de tratamentos estatísticos na série (Oliveira et al, 2010).

Com os períodos falhos identificados inicia-se o preenchimento das lacunas existentes, que, segundo Bertoni $\&$ Tucci (2002), procede-se em séries anuais, mensais ou, no máximo, séries quinzenais. A análise de consistência é a etapa posterior ao preenchimento das falhas e se caracteriza por verificar o grau de homogeneidade entre dados da estação de interesse e das estações vizinhas.

Grande parte das séries do Hidroweb da ANA possui porcentagem significativa de dados faltosos, contém poucos 
anos de dados, ou dados suspeitos. As falhas presentes nas series históricas das estações fluviométricas e pluviométricas dificultam a análise estatística dos dados. Quanto maior a porcentagem de dados faltosos, maior a probabilidade de que o valor máximo real não tenha sido medido. Séries pequenas podem não ser representativas e a presença de pontos atípicos pode indicar erros de medição (COSTA \& FERNANDES, 2015).

Enfim, é norma falhas nas séries históricas de dados, as quais devem ser identificadas e excluídas, devido aos estudos hidrológicos necessitarem de séries contínuas.

\section{Elaboração de mapa temático}

A Figura 2 apresenta a localização das estações pluviométricas e fluviométricas identificadas, e a Tabela 1 mostra a quantidade por bacia hidrográfica. Permitindo então a visualização dos postos hidrométricos pertencentes a cada bacia hidrográfica do estado da Paraíba.

Figura 2 - Estações hidrométricas com dados existentes

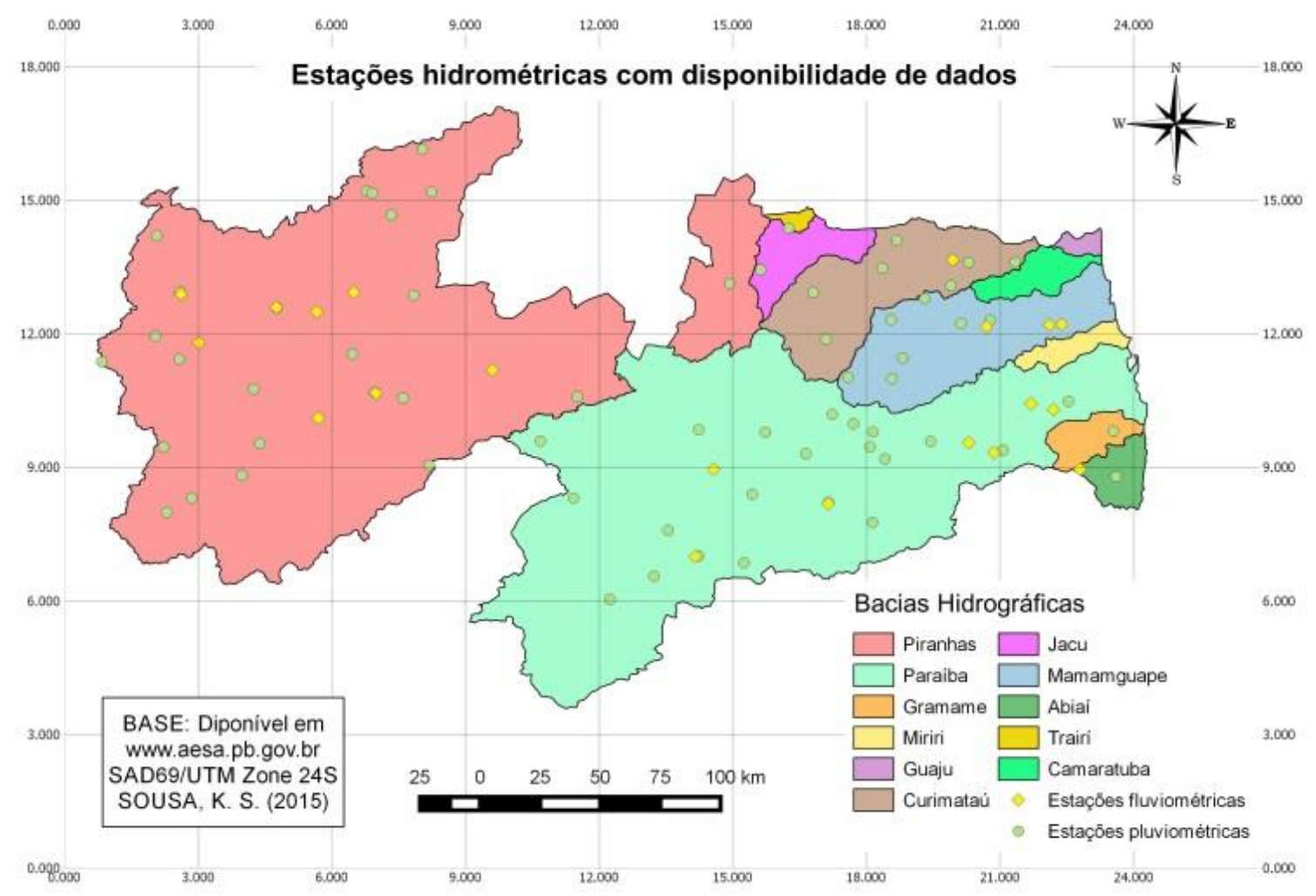

Fonte: Autoria própria.

Tabela 1 - Postos hidrométricos por bacias hidrográficas

\begin{tabular}{ccc} 
Bacia hidrográfica & $\begin{array}{c}\text { Postos } \\
\text { pluviométricos }\end{array}$ & $\begin{array}{c}\text { Postos } \\
\text { fluviométricos }\end{array}$ \\
\hline Piranhas & 32 & 8 \\
Paraíba & 27 & 7 \\
Gramame & 1 & 0 \\
Miriri & 0 & 0 \\
Guaju & 1 & 0 \\
Curimataú & 8 & 1 \\
Jacu & 1 & 0 \\
Mamanguape & 8 & 3 \\
Abiaí & 1 & 1 \\
Trairi & 0 & 0 \\
Camaratuba & 0 & 0 \\
\hline
\end{tabular}

Fonte: Autoria própria.

Pode-se observar que os postos hidrométricos identificados estão distribuídos de forma diferenciada em cada bacia hidrográfica.
As bacias do Rio Piranhas e Rio Paraíba possuem maiores quantidades de postos com dados disponíveis, enquanto que as bacias do Rio Miriri, Rio Trairi e Rio Camaratuba não possuem postos com dados de chuva e vazão disponíveis, as bacias do Rio Gramame, Rio Guaju e Rio Jacu possuem apenas uma estação pluviométrica e não possuem estação fluviométricas com dados existentes.

A existência de postos hidrométricos em uma bacia hidrográfica é de extrema importância para conhecimento dos processos hidrológicos do local, não apenas para estudos hidrológicos, mas também para avaliação do uso do solo, vegetação, entre outros (CALDEIRA, 2015).

\section{CONCLUSÕES}

Foram identificadas 79 estações pluviométricas e 20 estações fluviométricas com dados existentes na Paraíba, onde não estas se encontram distribuídas de forma diferente nas 11 bacias hidrográficas do estado, apresentando bacias sem dados de chuva e vazão disponíveis. 
Quanto às séries históricas de dados, os postos pluviométricos possuem 104 anos de medições, enquanto que os postos fluviométricos, 52 anos. Os dados de chuva encontram-se desatualizados, grande parte dos dados disponibilizados são anteriores ao ano de 2000. Foram identificadas falhas nos dados de chuva e vazão de todas as estações, compreendendo meses sem medições ao longo dos anos.

\section{REFERÊNCIAS BIBLIOGRÁFICAS}

AESA - Agência Executiva de Gestão das Águas do Estado da Paraíba. Disponível em: < http://www.aesa.pb.gov.br/geoprocessamento/geoportal/ shapes.html> Julho. 2015.

AGÊNCIA NACIONAL DE ÁGUAS. Inventário das Estações Fluviométricas. $2^{\circ}$ Edição. Brasília, 2009.

AGÊNCIA NACIONAL DE ÁGUAS. Orientações para consistência de dados pluviométricos. Superintendência de Gestão da Rede Hidrometeorológica. Brasília, 2012.

AGÊNCIA NACIONAL DE ÁGUAS. Orientações para operação de estações hidrométricas. Superintendência de Gestão da Rede Hidrometeorológica. Brasília, 2012.

ANA - Agência Nacional de Águas. Disponível em <http://www.ana.gov.br> Março. 2015.

ANA/ANEEL. Resolução Normativa Conjunta $\mathrm{N}^{\circ} 03$ de Agosto de 2010. Estabelece Estabelecer as condições e os procedimentos a serem observados pelos concessionários e autorizados de geração de energia hidrelétrica e dar outras providências.

BECKER, C.T.; MELO, M.M.; SILVA, M.; COSTA, M.N.M. Desempenho Temporal de Séries Pluviométricas no Estado da Paraíba: Uma Análise Comparativa. Anais Anais Workshop Internacional sobre Água no Semiárido Brasileiro, v. 1. Campina Grande, 2013.

BERTONI, J. C.; TUCCI, C. E. M. Precipitação. In TUCCI, C. E. M. Hidrologia: Ciência e Aplicação. 3 ed. Porto Alegre: Editora da UFRGS, 2002, 943p. (Coleção ABRH de Recursos Hídricos; vol. 4)

BRASIL. Lei $N^{\circ}$ 9.433, de 8 de Janeiro de 1997. Política Nacional dos Recursos Hídricos. Brasília, 1997.

CALDEIRA, T.L.; ARAÚJO, M.M.F.; BESKOW, S. Análise de Série Hidrológica de Precipitação no Sul do Rio Grande do Sul para aplicação na Gestão e Monitoramento de Recursos Hídricos. Anais IV Encontro Sul-Brasileiro de Meteorologia. 2015

COSTA, K.T.; FERNANDES, W.S. Avaliação do tipo de distribuição de probabilidade das vazões máximas diárias anuais do Brasil. Revista Brasileira de Recursos Hídricos, v. 20, n. 02, 442-451p, 2015.

ELESBON, A.A.A; SILVA, D.D.; SEDIYAMA, G.C.; MONTENEGRO, A.A.A.; RIBEIRO, C.A.A.S; GUEDES, H.A.S. Proposta Metodológica para Projeto de Redes Hidrométricas: Parte1-Espacialização não tendenciosa dos dados hidrológicos. Revista Brasileira de Engenharia Agrícola e Ambiental, v. 18, n. 9, 980985, Campina Grande, 2014.

GALINA, M.H.; VERONA, J.A. Fontes de Observações Meteorológicas no Estado de São Paulo. Revista Estudos Geográficos, v. 2(1). 107-118p, Rio Claro, 2004.

GOMES, M.G.; SANTOS, C.A.C.; SOUZA, F.A.S.; PAIVA, W.; OLINDA, R.A. Análise Comparativa da Precipitação no Estado da Paraíba Utilizando Modelos de Regressão Polinomial. Revista Brasileira de Meteorologia, v. 30, n.1, 47-58p, 2015.

IBGE - Instituto Brasileiro de Geografia e Estatística. IBGE: censo 2010, estados. Disponível em: <http://www.ibge.gov.br/estadosat/perfil.php?sigla=pb>. Acesso em: 24 de Junho de 2015.

IBIAPINA, A. V. et al. Evolução da hidrometria no Brasil. In: FREITAS, M.A.V. (Org). O estado das águas no Brasil. Brasília, DF: ANEEL, SIH; MMA, SRH; MME, 1999.

LEAL, A.C. Planejamento Ambiental de Bacias Hidrográficas como Instrumento para o Gerenciamento de Recursos Hídricos. Revista da Universidade Federal de Grande Dourados, ano 3, n.6, Dourados, 2012.

LUERCE, T.D.; OLIVEIRA, G.G.; GUASSELLI, L.A. Análise da distribuição espacial e temporal das chuvas aplicada ao estudo de cheias na bacia hidrográfica do rio dos Sinos/RS. Anais XV Simpósio Brasileiro de Sensoriamento Remoto. Curitiba, 2011.

OLIVEIRA, L. F. C. et al.; Comparação de metodologias de preenchimento de falhas de séries históricas de precipitação pluvial anual. Revista Brasileira de Engenharia Agrícola e Ambiental, v.14, n.11, p.11861192, 2010.

PARAÍBA. Caracterização Fisiográfica e Hidroclimática do Estado da Paraíba. Governo da Paraíba. João Pessoa, 2006.

PINTO, N.L.S; HOLTZ, A.C.T.; MARTINS, J.A.; GOMIDE, F.L.S. Hidrologia Básica. Editora Edgard Blücher, 1976.

SILVEIRA, J.F.; DUTRA, T.O.; PRIEBE, P. S.; SANTOS, W.C.; MILECH, R.N.; LAGOS, M.A.; REICHOW, C.; TAVARES, V.E. Q.; COLLARES, G. L.; MILANI, I. C. B.; NEBEL, A. L. C.; SUZUKI, L.E.A.S. Sistematização de Informações sobre Dados Hidrométricos do Complexo Lagunar Mirim-PatosMangueria. III Congresso Brasileiro de Oceanografia. Rio Grande do Sul, 2010.

TUCCI, Carlos E. M. Regionalização de Vazões. 1 ed. Rio Grande do Sul: Editora da Universidade Federal do Rio Grande do Sul, 2002. 256 p.

VILANOVA, M.R.N.; BALESTIERI, J.A.P. Qualidade dos Dados Fluviométricos obtidos através de Perfilamento Acústico. Revista Árvore, v. 37, n. 3, 531-538p, Viçosa, 2013. 